ISSN: 2773-7349

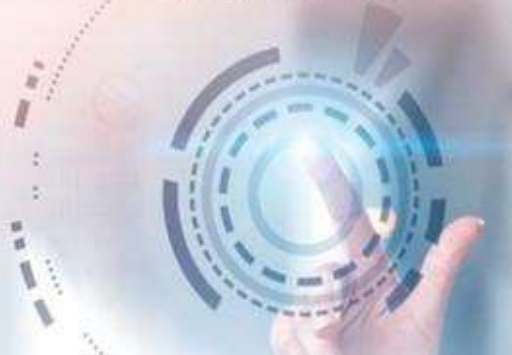

Sociedad \& Tecnología

Revista del Instituto Tecnológico Superior Jubones

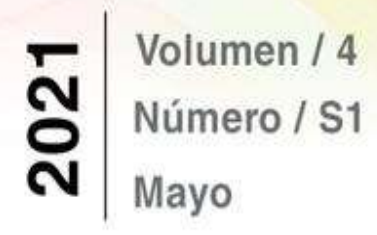

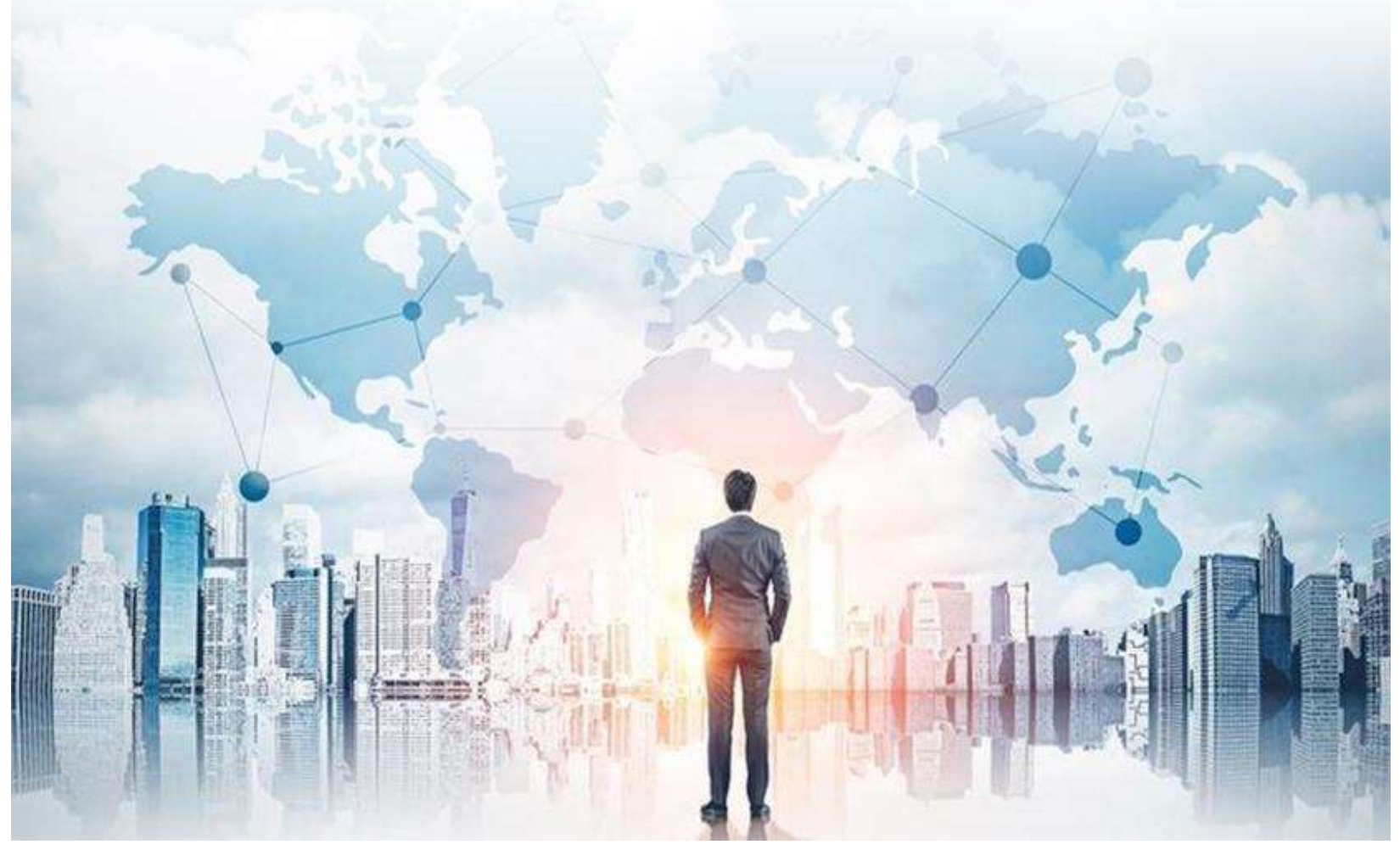




\section{El derecho a la salud de las personas con enfermedades catastróficas o degenerativas}

The right to health of people with catastrophic or degenerative diseases

Jaime Manuel Dutan Yunga ${ }^{1}$

E-mail: jdutan_est@utmachala.edu.ec

Orcid: https://orcid.org/0000-0002-7957-1564

Darwin Jeovanny Quinche Lavanda²

E-mail: dquinche@hotmail.com

Orcid: https://orcid.org/0000-0003-0660-4748

${ }^{1}$ Estudiante de la Maestría Derecho y Justicia Constitucional, Universidad Técnica de Machala.

${ }^{2}$ Profesor de la Universidad Técnica de Machala, Machala, Ecuador.

\section{Cita sugerida (APA, séptima edición)}

Dutan Yunga, J. M., Quinche Lavanda, D. Y. (2021). El derecho a la salud de las personas con enfermedades catastróficas o degenerativas. Revista Sociedad \& Tecnología, 4(S1), 256-271.

\section{RESUMEN}

El presente trabajo de investigación tiene por objetivo el análisis jurídico del acceso y justiciabilidad del derecho a la salud para las personas con enfermedades degenerativas o catastróficas, con énfasis en el caso del síndrome de Laron. Para su cumplimiento se realizó un estudio descriptivo con enfoque cualitativo de tipo revisión bibliográfica, sistematizado a través de los métodos analítico-sintético, exegético y de construcciones jurídicas. Los hallazgos evidencian fisuras en torno al acceso y justiciabilidad del derecho a la salud en Ecuador, en particular el derecho de las personas con este síndrome.

\section{Palabras claves:}

Acceso, justiciabilidad, derecho a la salud, enfermedades degenerativas

\begin{abstract}
The objective of this research work is the legal analysis of access and justiciability of the right to health for people with degenerative or catastrophic diseases, with emphasis on the case of Laron syndrome. For its fulfillment, a descriptive study was carried out with a qualitative approach of a bibliographic review type, systematized through analytical-synthetic, exegetical and legal constructions methods. The findings show fissures around the access and justiciability of the right to health in Ecuador, in particular the right of people with this syndrome.
\end{abstract}

\section{Key words:}

Access, justiciability, right to health, degenerative diseases 


\section{INTRODUCCIÓN}

El derecho a la salud es reconocido como derecho humano tanto en el Sistema Interamericano de los Derechos Humanos (SIDH) como en la legislación ecuatoriana, en la que se encuentra sistemáticamente relacionado con otros derechos constitucionales, entre los que destacan, la vida y la dignidad, así como la necesidad de recibir una protección especial del Estado que garantice su acceso igualitario y sin discriminación.

Este y otros derechos fundamentales de las personas, han sido socavados por la grave crisis política que ha sufrido el Estado ecuatoriano durante largo tiempo. En los años comprendidos entre el 1997 y el 2007, el escenario político ecuatoriano se caracterizó por la inestabilidad de gobernabilidad, golpes de Estado, derrocamientos y violencia social, contexto en el que las políticas públicas evidentemente no podían mantenerse o gozar de continuidad. Con la promulgación de la Constitución del 2008 y con el cambio de modelo de Estado, el Gobierno Central asumió a través del Ministerio de Salud Pública la responsabilidad de ser garante del derecho a la salud.

En tal sentido, la Corte Constitucional del Ecuador (CCE) ha reformado disposiciones legales, generando precedentes jurisprudenciales a favor de la actividad garantista del Estado que alcanzan el sector de la salud. Entre estas normativas se encuentra la Sentencia No. 679-18-JP/20 en el Caso No. 679-18-JP y otras acciones de protección acumuladas, planteadas al respecto de la justiciabilidad del derecho a la salud, en especial en lo referente al ejercicio de este derecho por parte de las personas con enfermedades degenerativas o catastróficas.

La garantía al acceso al derecho a la salud no se limita a la prestación de atención médica, además involucra otros elementos para que las personas recuperen, en lo posible, la salud; entre ellos la disponibilidad y acceso a los medicamentos, y en el caso particular de las personas con enfermedades degenerativas o catastróficas un trato especializado y oportuno. Sin embargo, no se ha alcanzado aún la satisfacción de las necesidades de todos los sectores que requieren la atención del Estado, especialmente a quienes la misma Carta Magna los reconoce como prioritarios, debido a su vulnerabilidad, en la práctica se observan limitaciones en la forma y las condiciones en que el Estado provee los servicios de salud.

Estas limitaciones han puesto sobre la mesa el debate público de la responsabilidad del Estado en la provisión de servicios médicos de calidad, que permitan la justiciabilidad del derecho a la salud y por lo tanto la tutela efectiva de los derechos de las personas a través de la implementación de políticas públicas que permitan el ejercicio de este derecho de forma plena.

Esta realidad ha motivado el presente estudio con el objetivo de realizar el análisis jurídico del acceso y justiciabilidad del derecho a la salud para las personas con enfermedades degenerativas 0 catastróficas, con énfasis en el caso del síndrome de Laron.

\section{Metodología}

Para el cumplimiento del objetivo propuesto se realizó un estudio descriptivo con enfoque cualitativo de tipo revisión bibliográfica, sistematizado a través de los métodos analítico-sintético para estudiar y resumir los hechos que permiten establecer el estado del arte del problema jurídico estudiado; la exegética para la mejor compresión, análisis, identificación y descripción de las leyes y normativas relativas a los hechos que generan efectos jurídicos en la justiciabilidad del derecho a la salud; y el método de construcciones jurídicas a través del cual se realiza el estudio de los problemas jurídicos que inciden en las restricciones formales $y$ materiales que impiden el acceso a un eficiente servicio a la salud a las personas con enfermedades degenerativas 0 catastróficas. 
La lógica indagatoria del estudio estuvo orientada a fundamentar teórica, doctrinal y jurisprudencialmente el derecho a la salud de las personas con estas enfermedades; determinar los estándares de protección de derechos humanos por parte del Estado; identificar las obligaciones del Estado en materia de protección de los derechos al Buen Vivir y determinar las obligaciones del Estado en lo relacionado a la protección del derecho a la salud de los grupos de atención prioritaria. Referentes sobre los cuales analizar la práctica del derecho a la salud en el Ecuador, con énfasis en las restricciones formales o materiales que impiden la justiciabilidad del derecho a la salud de las personas Síndrome de Laron.

\section{DESARROLLO}

\section{El derecho a la salud en el derecho constitucional ecuatoriano}

El Estado garantista de derechos tiene entre sus obligaciones controlar que las actividades que desarrollan las personas, ya sean naturales o jurídicas, públicas o privadas, no generen daños o menoscaben el derecho a la salud. Por tanto, corresponde a las personas el acceso a la prestación de servicios de salud con sujeción a los principios de equidad, universalidad, solidaridad, interculturalidad, calidad, eficiencia, eficacia, precaución y bioética, con enfoque de género y generacional de conformidad con lo establecido en el artículo 32 de la $\mathrm{CRE}$, sin perjuicio de la aplicación de otros principios como el de igualdad reconocido en la misma Carta Magna (Asamblea Nacional Constituyente del Ecuador, 2008).

La marcada diferencia en la capacidad de ejercer sus derechos y acceder a la prestación de servicios públicos, obliga al Estado a la emisión de políticas públicas y la adopción de acciones con el objeto de generar las mismas oportunidades de acceso a quienes por sus características o circunstancias particulares requieren un trato diferenciado, esto conforme a lo dispuesto por la CCE en la sentencia No.
080-13-SEP-CC dentro del caso No. 44511-EP (Corte Constitucional del Ecuador, 2010).

De estos presupuestos, se puede colegir que los derechos de los grupos de atención prioritaria reconocidos en la Constitución implican también un deber de protección reforzado por parte del Estado, tanto por las características propias de la relación de su condición con su derecho a la salud, como su relación con otros derechos como la igualdad. Esta protección y su justiciabilidad puede materializarse a través de prestaciones o abstenciones del Estado ecuatoriano desde la perspectiva del desarrollo de los derechos y garantías, la organización estatal y otros aspectos relevantes (Ayala, 2018).

Desde el marco normativo el Estado ecuatoriano tiene concebida el goce al derecho a la salud y su acceso por parte de personas con enfermedades degenerativas o catastróficas. Sin embargo, existen limitaciones con el suministro de medicamentos del cuadro nacional de medicamentos básicos (CNMB) como consecuencia del recorte presupuestario. Cabe entonces preguntarse ¿cuáles son los estándares de protección de los derechos humanos por parte del Estado?, ¿cuáles son las obligaciones del Estado en materia de protección de los derechos del Buen Vivir? y ¿cuáles son las obligaciones del Estado en lo relacionado a la protección del derecho a la salud de los grupos de atención prioritaria?

\section{Estándares de protección de derechos humanos por parte del Estado}

Las obligaciones de protección al derecho a la salud por parte del Estado están previstas en la Constitución en correspondencia a los instrumentos internacionales de Protección de Derechos Humanos reconocidos por el Ecuador. De esta forma, en el numeral 9 del art. 11 de la $C R E$, se señala que: "El más alto deber del Estado consiste en respetar y hacer respetar los derechos garantizados en la Constitución"; luego, los derechos humanos que conforman el bloque de 
constitucionalidad y desarrollados en la legislación ecuatoriana deben ser tutelados y garantizados por el Estado. Lo anterior se desprende de lo establecido en norma citada y en lo dispuesto en el art. 1 de la misma Carta Magna, en la que se reconoce al Estado ecuatoriano como un Estado Constitucional de Derechos y Justicia.

El reconocimiento como Estado garantista de los derechos humanos y el principio de supremacía constitucional implica, entre otras cosas, que la invocación de normas infra-constitucionales no puede ser utilizada para negar, restringir 0 menoscabar el ejercicio de estos derechos; por el contrario, de acuerdo a lo establecido en el numeral 2 del art. 11 y el art. 85 de la CRE corresponde al Estado la formulación, ejecución, evaluación y control de políticas y servicios públicos que garanticen el ejercicio de los derechos constitucionales. En este mismo sentido, la adopción de medidas de acción afirmativa que promuevan la igualdad real a favor de las personas que se encuentren en situación de desigualdad, como es el caso de las personas con enfermedades catastróficas o degenerativas tales como: cáncer, SIDA y el Síndrome de Laron.

Esta última enfermedad se caracteriza por la resistencia de la hormona del crecimiento generada por alteraciones del gen que codifica la proteína del receptor de dicha hormona, provocando lo que se conoce como enanismo (Núñez Almache et al., 2003). Resulta imposible negar la necesidad de tutelar derechos sobre la salud y vida de las personas con esta enfermedad, producto de una malformación congénita la cual afecta su ciclo de vida (Guevara, 2011).

En efecto, el Estado tiene la responsabilidad de formular y ejecutar políticas y servicios públicos para asegurar, promover $y$ garantizar el pleno goce de derechos; derivando beneficios especiales para las personas, sin distinción alguna, de acuerdo a los requerimientos sociales producto de su situación, sea por vulnerabilidad, debilidad o autonomía. Esto establece el reconocimiento de derechos, su exigibilidad y la prestación de servicios públicos, promoviendo la atención inmediata para tener una vida digna (Nogueira, 2017). En consecuencia, el Estado tiene el deber de evaluar y controlar toda disposición sobre políticas públicas para garantizar la protección de los derechos sociales. Estas disposiciones garantistas en gran medida actúan sobre prestaciones de servicios o bienes, que deben estar orientados a priorizar la atención en salud y asegurar entornos saludables para ofrecer mejores alternativas, conciliando algún derecho en conflicto.

Por tanto, el Estado se encuentra obligado a la protección prioritaria y especializada de las personas con enfermedades catastróficas 0 degenerativas, en los términos establecidos en el art. 50 de la CRE, que aluden al acceso a la atención especializada y gratuita en todos los niveles, de manera oportuna y preferente. Las responsabilidades ineludibles de protección del Estado, frente al modelo constitucional garantista de derechos, se sustentan en el ejercicio de funciones estatales para alcanzar la dignidad del ser humano, dotando de competencias a los tribunales de justicia en casos de vulneración de derechos. Así, por ejemplo, se establece uno de los mecanismos de protección como lo es la seguridad jurídica y su valor vinculante sobre la justicia correctiva y distributiva, al existir oposición entre la exigibilidad de derechos y la inacción estatal para satisfacer otros derechos, surgiendo una crisis de legalidad, sobre la fenomenología e ineficiencia de los poderes y control para ejercer un derecho (Ferrajoli, 1999, 2009).

La protección de los derechos humanos está relacionada directamente con el derecho a la tutela judicial efectiva, planteando aspectos relacionados al acceso a los tribunales para obtener una resolución motivada y argumentada amparada en la ley. La tutela judicial efectiva implica también que esta resolución sea de rápida exigibilidad y justiciabilidad, solventando la necesidad de un derecho, observando sustancialmente principios esenciales como: inmediación y celeridad, eliminando 
todo tipo de barrera existencial a nivel legislativo o procesal que dificulte su vigencia, provocando cambios necesarios en la realidad de exclusión y miseria, y fortaleciendo la función judicial en todo proceso que determinen derechos y obligaciones de cualquier orden (Ávila, 2009).

El Sistema Universal de Protección de los Derechos Humanos (SUDH), involucra el conjunto de normas sustantivas y procesales, así como los pronunciamientos de los órganos pertenecientes a la Organización de las Naciones Unidas (ONU), cuyo fin es la promoción y protección de los derechos humanos universales. EI SUDH está integrado por el Sistema de Tratados y el Sistema de Órganos de Derecho Humanos (VEONU, 2015). Estos instrumentos internacionales para la protección de los derechos humanos son firmados y ratificados voluntariamente por los Estados en ejercicio pleno de su soberanía. Esto deviene en su obligación de respeto al disfrute de los Derechos Humanos, impidiendo lesiones mediante la adopción de medidas y adecuación de su normativa interna.

Es así que la Corte Interamericana de Derechos Humanos (CIDH), ha manifestado, "Qué el método de interpretación previsto en las convenciones y tratados internacionales se acoge al principio de la primacía del texto, es decir que se deben aplicar criterios objetivos de interpretación" (Rossi \& Abramovich, 2007). Por tanto, el fin que persiguen los tratados y convenios internacionales es resguardar los derechos humanos $y$ prevenir su violación. Por este motivo se ha desarrollado inicialmente desde la jurisprudencia interamericana, la doctrina del Control de Convencionalidad, mirando la realidad social sobre las posibles vulneraciones de derechos.

De la misma forma, se entiende como control de convencionalidad el ejercicio que realiza la $\mathrm{CIDH}$ al analizar la complejidad de un asunto que ha sido puesto a su conocimiento, a través del cual se verifica la relación de la conducta del Estado y las disposiciones de la Convención, debiéndose explorar las circunstancias del caso (MacGregor \& Pelayo, 2012).

En este orden de ideas, García (2005), considera que el control de convencionalidad proporciona elementos indispensables para el respeto y garantía de los derechos fundamentales y sociales, con la implementación de recursos jurídicos para organizar el poder estatal, disponiendo el tribunal ordinario un control de las normas o recurriendo a otra instancia, haciendo efectivo un control concentrado, mayormente realizado por los tribunales constitucionales, para velar por la conformidad de las normas y leyes del Estado, en apego a los tratados y convenios en materia de protección de derechos humanos.

Luego, es oportuno enfatizar en la obligación que los Estados tienen de lograr la efectividad y legitimidad del ordenamiento interno, en correspondencia con los acuerdos de la Convención Americana sobre Derechos Humanos y el SIDH. Los Estados deben asegurarse de la protección de los derechos fundamentales, evitando incurrir en prácticas regresivas que terminen por violentar o limitar derechos sobre las personas, colectivos, comunidades, pueblos, nacionalidades (Rossi \& Abramovich, 2007; Convención Americana sobre Derechos, 1969).

En tal sentido, la ONU trabaja planteando objetivos para mejorar el nivel de vida y defender los derechos humanos, aprobando tratados y convenios internacionales para resolver conflictos de derechos aplicables en contra de inacción o falta de protección de un Estado, por ejemplo; la multiplicidad de elementos para comprender el derecho a la salud y la normativa legal dentro de cada Estado, adoptando deberes primordiales para los factores socioeconómicos que los afectan y proporcionando el medio adecuado para facilitar el acceso y justiciabilidad de los derechos fundamentales (Carbonell, 2009). 


\section{Obligaciones del Estado en materia de} protección de los derechos del Buen

\section{Vivir}

Entre los derechos del Buen Vivir, en términos de la CRE, se encuentran los de segunda generación, desarrollados en el marco de la prohibición de discriminación en relación con otros derechos, abordando obligaciones básicas y primordiales del Estado, tal es el caso del derecho a la salud, que implica, entre otras cosas, su aplicación y plena efectividad en emergencias sanitarias sobre sus bienes y servicios públicos. En este sentido, en caso de constatarse una restricción al mismo, será considerada una vulneración, que podría generarse a partir de la existencia de procesos administrativos ineficaces o por la falta de presupuesto designado al ejercicio de este derecho (Carbonell, 2009).

En la Carta Magna ecuatoriana, en su título VII, sobre el régimen del Buen Vivir, en su sección segunda, se reconoce al derecho a la salud como garantía y responsabilidad estatal, avizorando su carácter vinculante en ámbitos como: la seguridad social, los ambientes sanos, ciencia, gestión de riesgos y otros. Por consiguiente, el Estado está obligado a brindar el acceso permanente, oportuno y sin exclusión a programas, acciones y servicios de promoción y atención integral, mediante las instituciones de salud para mejor su condición de vida (Asamblea Nacional Constituyente del Ecuador, 2008).

En términos doctrinarios, la protección de los derechos humanos se traduce a través de las garantías que brinda el Estado a los derechos económicos, sociales y culturales, dentro de los cuales se encuentra reconocido el derecho a la salud como un derecho social básico (Rodríguez Maldonado, 2019). Este reconocimiento claramente está en contradicción con aquellas corrientes tradicionales que condicionan su protección y plena efectividad a una actividad prestacional de tipo económico, en otras palabras, a la existencia de recursos económicos (López, 2009).
En la misma línea de análisis, Holmes y Sunstein (2011), en su el libro El Costo de los Derechos, plantean la inexistencia de derechos en la medida que el ente regulador carezca de medios económicos para protegerlos. Esta idea exige del Estado la sostenibilidad y resguardo dentro de sus arcas de la recaudación de impuestos y otros ingresos que le permita ser garante de derechos; mostrando un Estado carente de virtualidad práctica, al negar el carácter exigible de los derechos sociales, sustentado en el elevado costo de aplicación de estos, pudiendo solo entenderse con la existencia de un servicio público, para brindar dichos beneficios en medida de su economía (Barrionuevo, 2021 y Chamba et al., 2021).

Estas corrientes tradicionales, que cuestionan la justiciabilidad directa de los derechos sociales, han encontrado criterios antagónicos, como los de Courtis y Ávila (2019), quienes ratifican que, para la protección de todos los derechos, independientemente de que sean de primera o segunda generación, se requerirá de una prestación, pero también una abstención por parte del Estado; citan para ello un ejemplo relacionado con el derecho a la propiedad, derecho de primera generación cuya satisfacción requiere erogaciones estatales cuantiosas. Esto evidencia que los derechos sociales, como el de la salud, no son los únicos que requieren una actividad prestacional para asegurar su protección, lo requieren todos, y no podría, en tal sentido, alegarse su imposible justiciabilidad en base a criterios eminentemente presupuestarios.

Como se ha expresado constitucionalmente existe una protección expresa, que procura garantizar a las personas que adolezcan enfermedades catastróficas 0 de alta complejidad, recibir atención prioritaria y especializada, de manera oportuna y preferente. Normativamente toda persona tiene derecho al disfrute del más alto nivel posible de salud, física y mental en relación con los derechos humanos, en nuestra Constitución esto es parte del Buen Vivir o Sumak Kawsay, lo que involucra la vida en plenitud, obligando al Estado a reorganizar 
el uso de sus recursos en la implementación de políticas públicas que garanticen una vida digna, salud y medicamentos de calidad.

\section{Obligaciones del Estado en lo relacionado a la protección del derecho a la salud de los grupos de atención prioritaria}

Se debe tener presente que históricamente los grupos de atención prioritaria se han reconocido por su condición de riesgo, lo que implica que el Estado deba prestar especial protección a personas en condiciones de doble vulnerabilidad, para que puedan acceder a mejores condiciones de vida, en los términos previstos en el artículo 35 de la CRE. En este sentido, la Constitución hace énfasis en la atención a los niños, niñas y adolescentes que, por nacer con una alteración genéticamente modificada, presentan alteraciones físicas, novedosas en el ámbito de la medicina, estimulando una nueva necesidad para acceder a la salud y obligación estatal (Asamblea Nacional Constituyente del Ecuador, 2008).

Bajo esta premisa, es importante destacar que, si bien no debe equipararse a las enfermedades catastróficas con discapacidades, debe considerarse que varias enfermedades catastróficas, como el Síndrome de Laron, generan discapacidades al impedir el normal desenvolvimiento físico. En este sentido, la Ley de Discapacidades se fundamenta en principios de no discriminación de las personas con discapacidad y de la acción afirmativa sobre la atención especializada que deben percibir estos grupos vulnerables por parte del Estado, ya sea de forma individual o grupal (Asamblea Nacional del Ecuador, 2012).

Esta atención garantista, sustentada en las obligaciones constitucionales referidas, requiere ser cumplida por las instituciones públicas, sobre las acciones de carácter prestacional en salud. Lastimosamente, la práctica no es consecuente con esta obligación constitucional, el servicio de salud carece de celeridad y eficiencia, fraccionando derechos y provocando vulneración o limitación al acceso integral a la salud, inobservando la obligación estatal en decisiones judiciales (Silva, 2009).

La ONU ha venido trabajando por los derechos humanos dando así una especial atención a los grupos más vulnerables de la sociedad, específicamente por su condición física, psicológica, social o cultural, prestando especial atención a las personas vulnerables (Alejandro et al., 2018; Novillo, 2019 y Serrano et al., 2019).

En el caso puntual del derecho a la salud, este no debe contemplar una mera acción de atención médica; esto implica libertades y derechos proyectados en una singular garantía sobre el derecho a un sistema de protección de salud, en igualdad de condiciones y sin discriminación. Consecuentemente los principios de eficacia, universalidad y solidaridad, imponen al Estado la obligación de extender su protección a los derechos sociales, ampliando el campo de acción al reconocimiento de prestación de servicios de las instituciones privadas, que contribuyan en la accesibilidad y adquisición de medicamentos, eliminando brechas para que dicho derecho no sea lesionado, o amenace la protección garantista desde la perspectiva legal (Arango, 2009).

Esto origina una protocolización de normas administrativas, sobre la necesidad de poder contar con un convenio de compras urgentes para insumos médicos. Al no existir otra alternativa médica en el mercado farmacéutico del país, resulta necesario el proceso de evaluación y control de políticas públicas respecto a los bienes que necesariamente son aplicables para reparar o reintegrar la salud de un paciente.

Por tanto, muchas políticas públicas del Estado, como aquellas generadas por Colombia, han optado por evaluar el alcance de este derecho en sede judicial para evitar la vulneración de este tipo de derechos fundamentales dentro del sistema de prestación de servicios a la salud y demás derechos sociales (Corte 
Constitucional Colombiana, 2008; López, 2009). En el caso argentino, la prestación del servicio de salud ha contemplado reformas constitucionales para otorgar beneficios sociales, dotándolas de carácter integral e irrenunciabilidad, sobre un seguro social obligatorio, pero no como una garantía expresa al derecho a la salud, sino a una cobertura de contingencias sociales, para la libre contratación de mejores prestaciones en salud, autofinanciadas por instituciones privadas (Abramovich \& Pautassi, 2008; Bazán, 2017).

Desde lo individual, las personas tienen derecho a que el medicamento o prestación de servicio contribuya al más alto nivel posible de salud y desde lo colectivo, la disponibilidad y el acceso de medicamentos deben contribuir al marco de una política pública de salud basada en derechos fundamentales, predominando los intereses de la salud pública por sobre los intereses económicos, comerciales o particulares, conforme lo dispuesto en el artículo 363 de la CRE. Dicho artículo prevé que el Estado será responsable de garantizar la disponibilidad y acceso a medicamentos de calidad, seguros $y$ eficaces, regular su comercialización y promover la producción nacional y la utilización de medicamentos genéricos que respondan a las necesidades epidemiológicas de la población (Asamblea Nacional Constituyente del Ecuador, 2008).

El análisis del derecho a la salud, a la luz de la ley, la doctrina y la jurisprudencia, permite reconocer su importancia y su relación en el ejercicio de otros derechos constitucionales; en este sentido, destaca que estos derechos que pudieran verse afectados por la falta de atención oportuna y especializada a las personas, especialmente de aquellas con enfermedades catastróficas. La obligación prestacional de servicios de salud obliga al Estado a propiciar políticas públicas, así como medidas de acción afirmativa, para garantizar la recuperación de su salud. En aplicación el principio pro homine, el derecho a la salud deberá materializarse mediante cualquier método clínico, científico, natural o ancestral, que conlleve a la persona afecta a alcanzar su más alto nivel de beneficio, eliminando barreras presupuestarias o administrativas.

Así en el caso del Síndrome de Laron, catalogado como enfermedad huérfana o raro, cuya solución médica no se encuentra clínicamente establecida, es preciso que el Estado garantice las investigaciones direccionadas a lograr la mejoría de las sometidas que padecen de esta enfermedad (Guevara, 2011).

Después del análisis realizado sobre el marco doctrinal y jurisprudencialmente del derecho a la salud de las personas con enfermedades catastróficas o degenerativas se impone el acercamiento reflexivo a la realidad ecuatoriana.

\section{Práctica del derecho a la salud en el Ecuador}

Al tratar el derecho a la salud y su reconocimiento en el sistema jurídico nacional e internacional, es importante destacar sus alcances a fin de no interpretarlo como el ideal de estar sano que podría considerar en muchos casos como utópico e irrealizable. Lo anterior se desprende de la realidad innegable de que por razones genéticas muchas personas se encuentran en predisposición de enfermarse o desarrollar patologías que comprometen su estado de salud, unas en mayor medida que otras.

De lo expuesto, el tratadista Figueroa (2013), afirma que el análisis del derecho a la salud podría entenderse como la obligación de cuidado y protección que debe brindar el Estado, y por su parte, el derecho a las personas de beneficiarse de este deber que, abraca el cuidado a la previsión y control del estado de salud, la distribución de recursos, medicinas y demás elementos necesarios para conservar la salud.

En el caso ecuatoriano, se concibe la existencia de cuadros básicos de medicamentos para el tratamiento de enfermedades degenerativas, a través de los cuales no se permite la recuperación integral de la salud de las personas que 
acuden a la prestación de este servicio público. Debe destacarse que, el Estado está obligado a la formulación de políticas públicas que permitan la universalización de la salud, de tal forma que amplíe su cobertura, hecho que se encuentra limitado en relación a la restricción de la adquisición de medicamentos por el cuadro básico emitido por el Ministerio de Salud. Lo expuesto ya ha sido observado por la CCE en las sentencias No 364-16-SEP-CC, dictada dentro del caso N. ${ }^{\circ} 1470-14-E P$, y No. 25-20-IS/20 dictada dentro del caso No. 25-20-IS (Corte Constitucional del Ecuador, 2016, 2020).

Pese a la existencia de jurisprudencia vinculante emitida por la Corte Constitucional en la materia, a las disposiciones constitucionales y legales vigentes y al reconocimiento del derecho a la salud como fundamental en los instrumentos internacionales, la prestación del servicio de salud no ha protegido a las personas con enfermedades catastróficas de la marginación, exclusión, y discriminación de las que son objeto (Tinoco Noblecilla, 2018; Morales Llanganate, 2018). En el caso de síndrome de Laron, al ser una enfermedad de alta complejidad por alterar la salud de quien la padece, requiere de atención oportuna y especializada; sin embargo, se destaca la ausencia de protección necesaria en la prestación de servicios públicos, tanto en el cuidado de su salud y el acceso a medicamentos adecuados (Jiménez Barbosa et al., 2017; Mosquera, 2017;).

En el caso del Síndrome de Laron, es necesario brindar un tratamiento especial a los niños(as) que lo padecen y que día a día se ven envueltos en la desesperanza al no poder contar con la medicina necesaria, situación ocasionada por muchos factores socioeconómicos y más aún por políticas públicas limitadas en el acceso a la salud.

Esta situación quedó claramente evidenciada en la sentencia del 1 de diciembre de 2010, dictada por el Tribunal Segundo de Garantías Penales de Pichincha dentro de la acción de protección N. ${ }^{\circ} 139$ 2010, presentada por los representantes de niños que padecen del Síndrome de Laron contra del Ministerio de Salud Pública del Ecuador al no tener el medicamento necesario para poder brindar un tratamiento especial a dichos niños, ya que el medicamento no está clasificado dentro del cuadro de medicamentos básicos.

Esta omisión genera la vulneración de derechos constitucionales establecidos en el artículo 11 numeral 2, 4, 6, 9, además de los artículos 33 y 35, haciendo énfasis en el derecho a la salud de personas; además, evidencia la inacción estatal en la atención de este tipo de enfermedad, causando y provocando daños graves en las personas que padecen el síndrome. Es por ello que, los representantes de los menores con Síndrome de Laron, exigen la protección constitucional de sus derechos, para que se pueda declarar al síndrome, parte de enfermedades catastróficas 0 de alta complejidad, lo cual permitiría solicitar al Estado el medicamento necesario.

La CCE, para poder resolver esta cuestión, analizó el derecho y sus implicaciones en cinco acápites temáticos y un acápite final de los casos acumulados, detallando lo siguiente:

1. El derecho a la salud, prevención de enfermedades y promoción del derecho a la salud.

2. El derecho al acceso a medicamentos de calidad, seguros y eficaces, para el mejor disfrute.

3. El derecho de las personas al acceso a la información y consentimiento informado.

4. El derecho a la tutela judicial efectiva en relación al acceso a medicamentos.

5. Indicadores para valorar el derecho a la disponibilidad y acceso a medicamentos.

6. Derecho a medicamentos de calidad, seguros y eficaces en casos conocidos por la Corte. 
Esta situación ha provocado la emisión por parte de la CCE la sentencia, $\mathrm{N}^{\circ} 679-18-$ $\mathrm{JP} / 20$, en la que se resuelve llamar la atención al gobierno nacional por la regresividad en la determinación del presupuesto de salud; asimismo, dispone la formulación de políticas públicas por los organismos estatales sobre las necesidades médicas de los medicamentos utilizados en el tratamiento de enfermedades catastróficas, aduciendo la existencia de un limitado stock de medicinas y la situación de algunos pacientes que no tienen acceso a la medicina específica y esencial para su tratamiento médico y recuperación, al no estar presentes en el CNMB. Esta sentencia comprendía aspectos inejecutables al ordenar la adquisición de medicamentos que al momento no contaban con registro sanitario; lo anterior, comprende un requisito sine qua non para la adquisición y comercialización de medicinas en Ecuador.

Se debe destacar que la vulneración del derecho constitucional a salud por parte del Ministerio de Salud Pública ha sido declarada y reconocida en la acción de protección N.o 139-2010, requiriendo incluso que la CCE emita la sentencia No 364-16-SEP-CC en relación al incumplimiento de lo dispuesto como medias de reparación a través de las cuales se pretendía resarcir los efectos generados por la falta de atención a las personas con Síndrome de Laron (Corte Constitucional del Ecuador, 2016); sin embargo, a pesar de que se ha recurrido al máximo órgano de control constitucional los derechos de este grupo de atención prioritaria aún no ha sido atendido conforme lo establece la CRE, esto es, a través de un servicio de salud especializado y oportuno.

Conforme a las evidencias aportadas en los documentos del proceso constitucional, existen problemas en el gasto público y la ejecución presupuestaria a cargo del Ministerio de Salud Pública del Ecuador, generando deficiencias en la sostenibilidad de este derecho social. En la sentencia objeto de análisis se ordena al Estado a adoptar medidas afirmativas que, en conjunto con las políticas públicas, permitan la adquisición de medicamentos de calidad que incidan positivamente en la salud de los accionantes.

Es necesario destacar que la sentencia precisa que los medicamentos a adquirirse deberán ser seguros y eficaces, sin embargo, la ausencia de protocolos, estudios y demás actos administrativos para establecer la validez de los medicamentos, impiden la ejecución inmediata de lo resuelto. Por otra parte, las omisiones del Ministerio de Salud Pública al momento de reglamentar sus actuaciones, han impedido el efectivo ejercicio de este derecho, por lo que se ha requerido utilizar a la justicia como medio de protección para acceder a un tratamiento de manera inmediata. A pesar de lo dispuesto por la CCE la mencionada protección no ha podido ser obtenida, ya que para poder incorporar al sistema de la red de salud pública esta enfermedad tiene que ser evaluada con relación al riesgo de muerte que pueda sufrir el paciente, para desde allí empezar a protocolizar los métodos de diagnósticos en las instituciones del Ministerio de Salud, incluido los organismos de control y vigilancia.

El Pacto Internacional de Derechos Económicos, Sociales y Culturales considera el derecho a la salud como un deber del Estado, el que debe ser atendido progresivamente; sin embargo, en el caso ecuatoriano, se destaca la necesidad de la intervención de la justicia constitucional para la justiciabilidad del derecho a la salud, hecho que impide el acceso oportuno a este servicio público, especialmente a las personas que pertenecen a grupos de atención prioritaria (Ministerio de Relaciones Exteriores Defensa y Seguridad Pública, 1969).

La violación de derechos descrita, requiere la incorporación de políticas públicas al sistema de la red de salud pública, por lo que dicha enfermedad tiene que ser declarada con relación al riesgo de muerte que deba sufrir el paciente, para desde allí empezar a protocolizar los métodos de diagnósticos en las instituciones del Ministerio de Salud, mediante los organismos de control y vigilancia. 
En atención a los antecedentes expuestos, así como a elementos doctrinarios y jurisprudencias con relación a la aplicación de la CRE y los instrumentos internacionales, se puede colegir que el Estado no ha cumplido su obligación de tutelar, garantizar, proteger, respetar y hacer respetar los derechos de las personas con enfermedades catastróficas en lo que corresponde a la atención oportuna y especializada. Destaca que, el reconocimiento de las enfermedades catastróficas, como el Síndrome de Laron, no ha sido por la emisión de políticas públicas, sino por las decisiones judiciales que han puesto en evidencia la omisión de las obligaciones del Estado.

Si bien la Corte Constitucional del Ecuador a través de las sentencias analizadas ha marcado un camino importante que reconoce la vulneración de los derechos de las personas con enfermedades degenerativas, como el Síndrome de Laron; comprende también su obligacion la de establecer directrices que obliguen al Ministerio de Salud Pública y al Ministerio de Finanzas, la planificacion presupuestaria adecuada que permita la atención integral, oportuna y especializada de estos grupos de atención prioritaria. Además, el Ministerio de Salud Pública debe emitir protocolos para la atención de urgencias y emergencias relacionadas a las personas 0 grupos de atención prioritaria.

\section{CONLUSIONES}

El desarrollo de la investigación y el cumplimiento de los objetivos propuestos, permite concluir lo siguiente:

- El derecho a la salud de las personas con enfermedades degenerativas o catastróficas comprende un deber de protección reforzada por parte del Estado, que implica no solo la prestación de un servicio básico, sino de un servicio oportuno eficiente $y$ especializado que permita el tratamiento de las diferentes afecciones de las personas que pertenecen a grupos vulnerables. El derecho a la salud, especialmente de las personas con enfermedades degenerativas, debe atenderse bajo la perspectiva de la accesibilidad de una serie de elementos que lo componen, esto es, bienes y servicios, incluido los medicamentos, que permitan alcanzar el más alto nivel de salud posible.

- El servicio de salud brindado a personas con enfermedades degenerativas o catastróficas por el Estado ecuatoriano no ha concebido a las diferentes enfermedades catatróficas limitando el acceso al derecho a salud de personas con afecciones, como es el caso de los que padecen el Síndrome de Laron, la atención especializada, medicamentos, y otros servicios que le permitan el restablecimiento de salud o al menos un nivel de vida digna, correspondiendo a una responsabilidad del Estado cuya protección ya ha sido ordenada por la justicia constitucional a través de precedentes jurisprudenciales.

- Las dispocisiones normativas infra constitucionales, y los precedentes jurisprudenciales de la justicia constitucional, no han permitido la justiciabilidad del derecho a la salud de las personas con enfermedades degenerativas o catastróficas, debido a la multiplicidad de elementos restrictivos y actos burocráticos que se requieren para la implementación de servicios que permitan la atención de las diferentes enfermedades degenerativas que se presentan en los centros de salud.

- La justicia constitucional no ha sido suficiente para la protección de los derechos, puesto que la discrecionalidad administrativa del Ministerio del Salud Pública del Ecuador, fracturando así el ejercicio de las competencias establecidas en la Constitución y la Ley.

- La justiciabilidad del derecho a la salud de las personas con enfermedades 
castróficas, como es el caso de las personas con Síndrome de Laron, requiere de la implementación de políticas públicas dirigidas a la especialización de los servicios, tanto en el recurso humano como en la provisión de otros bienes y servicios que sean necesarios para el tratamiento que requieren este tipo de enfermedades. Debido a la especial condición de estas personas, cuya condición no ha sido prevista por el Ministerio de Salud Pública del Ecuador se requiere la implementación de medidas de acción afirmativa que permitan de forma mediata e inmediata el acceso a medicamentos que no se encuentren dentro del cuadro básico, y que hubieran obtenido resultados positivos en otros centros de salud internacional a los cuales el Estado tenga acceso por las diferentes redes de salud a las que pertenece.

\section{REFERENCIAS BIBLIOGRÁFICAS}

Abramovich, V., \& Pautassi, L. (2008). El derecho a la salud en los tribulaes. Algunos efectos del activismo judicial sobre el sistema de salud en Argentina. Salud Colectiva, 263264.

Alejandro Contento, K. J., Erráez Alvarado, J. L., Vargas Gaona, M. D. C., \& Espinoza, E. (2018). Consideraciones sobre la educación inclusiva. Revista Metropolitana de Ciencias Aplicadas, 1(3), 18-24.

Arango, R. (2009). La jurisdicción social de la tutela. En: C. C. Ávila, La protección de los derechos sociales (pp. 311-313). Quito: V\&M Gráficas.

Ávila, R. (2009). Los retos en la exigibilidad de los derechos del buen vivir en el derecho ecuatoriano. En: C. Courtis, \& R. Ávila, La protección judicial de los derechos sociales (pp. 543-549). Quito: V\&M Gráficas.
Ayala, E. (2018). Rasgos históricos. En: E. Ayala, Evolucion Constitucional del Ecuador. Quito: Universidad Andina Simón Bolivar/ Corporación Editora Nacional.

Barrionuevo Núñez, J. L. (2021). El efecto del teletrabajo en el empleo en Ecuador durante la crisis sanitaria 2019-2020. Sociedad \& Tecnología, 4(2), 223-234. https://doi.org/10.51247/st.v4i2.1

06

Bazán, V. (2017). La protección de los Derechos a la Salud y la Seguridad Social en Argentinall, Revista Jurídica de los derechos sociales, Universidad Pablo Olavide, Sevilla. Lex Social, 7(1) https://www.upo.es/revistas/index. php/lex_social/article/view/2385

Carbonell, M. (2009). El derecho a la salud en el derecho internacional de los derechos humanos. En: C. Curtis, \& R. Ávila, La proteccion judicial de los derechos sociales (pp. 173-200). Quito : V\&M Gráficas.

Courtis, C., \& Ávila, R. (2009). La protección de los derechos sociales en la jurisprudencia constitucional ecuatoriana. En: C. Courtis, \& R. Ávila, La protección judicial de los derechos sociales (pp. 617-673). Quito: V\&M Gráficas.

Chamba Bernal, J. L., Bermeo Cuenca, L. A., \& Campuzano Vásquez, J. A. (2021). Variables determinantes en el crecimiento económico del Ecuador función Cobb-Douglass 2007-2019. Sociedad \& Tecnología, 4(2), 109-122. https://doi.org/10.51247/st.v4i2.9 8

Ferrajoli, L. (1999). Derechos y garantías. Madrid Ferraz: trotta.

(2009). Definición del concepto de los derechos fundamentas. En: L. Ferrajoli, Los fundamentos de los 
derechos fundamentales (pp. 1924). Madrid: TROTTA.

Figueroa, G. R. (2013). El derecho a la salud. Santiago de Chile. Estudios constitucionales 11(2). Doi: http://dx.doi.org/10.4067/S071852002013000200008

García, A. (2005). El control de la constitucionalidad en nuestro sistema jurídico. Derechoecuador.com, https://www.derechoecuador.com/ el-control-de-la-constitucionalidaden-nuestro-sistema-juriacutedico.

Guevara, A. J. (2011). Una comunidad ecuatoriana podría tener la clave contra el cáncer. Semana. https://www.semana.com/unacomunidad-ecuatoriana-podriatener-clave-contra-cancer/235671$3 /$

Holmes, S., \& Sunstein, C. (2011). Por qué la libertad depende de los impuestos. En: S. Holmes, \& C. Sunstein. El costo de los derechos. Buenos Aires: siglo veintiuno.

Jiménez Barbosa, W. G, Granda Kuffo, M. L, Ávila Guzmán, D. M, Cruz Díaz, L. J, Flórez Parra J. C, Mejía, L. S, Vargas Suárez, D. C. (2017). Transformaciones del Sistema de Salud Ecuatoriano. Rev Univ. Salud,19(1), 126-139. DOI: http://dx.doi.org/10.22267/rus.171 901.76

López, D. (2009). El derecho fundamental a la salud y el sistema de salud: los dimeas entre la jurisprudencia, la economia y la medicina. En: C. Courtis, \& R. Ávila, La protección judical de los derechos sociales (pp. 386-389). Quito: V\&M Gráficas.

Mac-Gregor, E., \& Pelayo, C. (2012). La obligación de "respetar" y "garantizar" los derechos humanos a la luz de la jurisprudencia de la corte interamericana. Santiago. Estudios constitucionales, 10(2).
Morales Llanganate, T. (2018). Las políticas públicas y las enfermedades catastróficas. (Tesis de Grado). Universidad Regional Autónoma de los Andes "UNIANDES"

Mosquera, D. (2017). Síndrome de Laron. Ecuador tiene la mayoría de casos en el mundo. Edicion Medica del Ecuador.

https://www.edicionmedica.ec/secc iones/salud-publica/s-ndrome-delaron-ecuador-tiene-la-mayor-a-decasos-en-el-mundo-89570

Nogueira, H. (2017). La protección convencional de los Derechos de los Niños y los estándares de la Corte IDH sobre medidas especiales de protección por parte de los Estados Partes respecto de los niños, como fundamento para asegurar constitucionalmente los Derechos de los niños. Talca. Ius et Praxis.23(2).

Novillo, L. (2019). La inclusión de los grupos de atención prioritaria en proyectos de vinculacion de la sociedad. Cienfuegos. Conrado, 15(67).

Núñez Almache, O., Rojas Gabulli, M., Falen Boggio, J., Del Aguila Villar, C., \& Lu de Lama, R. (2003). Descripción de un caso de resistencia a la hormona del crecimiento. Diagnostico, 42(4), Julio-Agosto.

Rodríguez Maldonado, M. (2019). La judicialización del acceso a medicamentos para enfermedades catastróficas y de alta complejidad en el Ecuador. Un estudio de los casos relevantes de la Corte Constitucional 2014 - 2017. (Tesis de Maestría). Universidad Andina Simón Bolíva. Sede Ecuador

Rossi, J., \& Abramovich, V. (2007). La tutela de los derechos económicos, sociales y culturales en el artículo 26 de la Convención Americana sobre Derechos Humanos. Estudio Socio- 
Juridico.,Bogotá (Colombia),
9(Número especial), 34-53.

Silva, C. (2009). Los derechos sociales y el desafío de la accion de protección. En: C. Courtis, \& R. Ávila, La protección judicial de los derechos sociales (pp. 603-610). Quito: V\&M Gráficas.

Serrano Polo, O. R., Espinoza, E., \& Guamán, E. E. (2019). La inclusión en la enseñanza básica ecuatoriana. Revista Metropolitana de Ciencias Aplicadas, 2(2), 69-74.

Tinoco Noblecilla, M. (2018). La Salud como Servicio Público: Un reto ante la vulneración del derecho a la salud de personas con enfermedades catastróficas y su acceso a medicamentos. (Trabajo de titulación previo a la obtención del título de abogada de los tribunales y juzgados de la República del Ecuador). Universidad Católica de Santiago de Guayaquil.

VEONU. (2015). El Sistema Universal de Protección de los Derechos Humanos. Examen Periódico Universal, Todas las personas. https://www.examenonuvenezuela. com/examen-veonu/ddhh.

\section{Fuentes normativas.}

Ecuador. Asamblea Constituyente. (20 de octubre de 2008). Constitución de la República del Ecuador. Montecristi:
Registro Oficial No. 449. Quito. Ecuador.

Asamblea Nacional. (25 de septiembre de 2012). Ley Orgánica de Discapacidades. Quito: Suplemento del Registro Oficial No. 796. Quito. Ecuador.

Convención Americana sobe Derechos Humanos. (22 de noviembre de 1969). Pacto de San José de Costa Rica. Registro Oficial No. 801. Quito. Ecuador.

Ministerio de Relaciones Exteriores Defensa y Seguridad Pública. (24 de enero de 1969). Pacto Internacional de Derechos Económicos, Sociales y Culturales. Registro Oficial No. 101. Quito. Ecuador.

\section{Fuentes Jurisprudenciales:}

Corte Constitucional Colombiana (4 de marzo de 2008). Sentencia No. T238/2008, T-1614078.

Corte Constitucional del Ecuador (15 de diciembre de 2010). Sentencia No. 080-13-SEP-CC dentro del caso, 445-11-EP.

Corte Constitucional del Ecuador (15 de noviembre de 2016). Sentencias No 364-16-SEP-CC, 1470-14-EP.

Corte Constitucional del Ecuador (12 de agosto de 2020). Sentencia No. 2520-IS/20, 25-20-IS.

Limitaciones y estudios futuros: La principal limitación del estudio está en el tipo de investigación que circunscribe su alcance al análisis del objeto de estudio sin establecer relaciones entre las variables.

Los autores se proponen continuar esta misma línea de estudio, para profundizar en las causas y efectos de la vulneración del derecho de salud del pueblo ecuatoriano.

Reconocimiento: Se reconoce el apoyo brindado por los profesores de la Maestría Derecho y Justicia Constitucional de la Universidad Técnica de Machala; la calidad de Ios contenidos impartidos que han permitido en gran medida la elaboración de este trabajo. 
Contribución de los coautores: Ambos autores participaron activamente en la elaboración del presente trabajo. El maestrante Dutan Yunga tuvo a su cargo la búsqueda, recuperación, estudio y procesamiento de los documentos, y la elaboración de la base de datos, por su parte el profesor Darwin Jeovanny sirvió como asesor y guía, además de participar junto al otro coautor en la construcción del discurso escrito y su revisión final. 